\title{
UMA POLITICA INTEGRADA DE SAÚDE PARA O ESTADO DE SÃO PAULO, BRASIL
}

\author{
Ernesto Lima Gonçalves * \\ Evelyn Naked Castro Sá** \\ Gastão E. de Arruda Camargo *** \\ João Yunes**** \\ Otávio Mercadante ** \\ Ruy M. Gomes Pinto $* * * * *$ \\ Sergio Reis Quaglia****** \\ Tristāo Pereira da Fonseca F:*** \\ Zilah Wendel Abramo**
}

\begin{abstract}
LIMA Gonçalves. E. et al. - Uma politica integrada de saúde para o Estado de São Paulo, Brasil. Rev. Saúde públ., S. Paulo, 9:169-79, 1975

Rescmo: Proposta de uma politica de saude para atender à população do Estado de São Paulo, Brasil com base num esquema de integração de recursos. com triplice alcance. Em primetro lugar, a integração entre as atuvidades de medina preventiva e as de sentido curativo e assistencial. Em segundo lugar, a integração dos setores que executam os programas de saneamento básico, sob voordenação da "autoridade santária" representada jela Secretaria de Estado da Saide. Em terceiro lugar, a integração dos órgãos e estruturas assistenciais - ambulatorials ou hospitalares - tanto as ligadas wo poder público federal, estadual e municipal. amanto as dependentes da matatua varticular, num "sistema de saúde".
\end{abstract} Suide.

Unitermos: Saúde, integração de recursos. São Paulo, Brasıl. Sistema de

t. consideração dos problemas de saúde que a comunidade enfrenta no Estado de São Paulo e a participação que o poder público e a iniciativa particular derem ter na sua solução. pode ter como ponto de partida a definição do objetivo que se pretende atingir. Pretendemos usar aquí a conceituação da Organização Mundial da Saúde em que se afirma que "saúde é o estado de completo bem-estar físico. mental e social do homem".

Trata-se de conceito que, à primeira rista. pode parecer exageradamente abrangente: contudo. apenas o cidadão que se

* Da Faculdade de Medicina da Lniversidade de São Paulo - Av. Dr. Arnaldo, 455 São Paulo, SP - Brasil

** Da Secretaria de Estado da Saúde - Av São Luı. 99 - São Paulo, SP — Brasil

*** Da Secretaria de Higiene e Saúde da Prefeitura do Municípı de São Paulo - Hospital Municipal - Rua Castro Alves, 60 - São Paulo, SP - Brasil

**** Da Faculdade des aúde Pública da Lniversidade de São Paulo — Av. Dr. Arnaldo, 715 - São Paulo, SP - Brasil

*****: Da Faculdade de Arquitetura e Urbanımo da Universidade de São Paulo - Cidade Universitária Armando Salles de Oliveira - São Paulo. SP - Brasıl

******* Do Instituto de Assistênela Médica do Servidor Público Estadual - Rua Pedro de Toledo. 1800 - Sāo Paulo. SP - Brasıl 
LIMA GONÇALVES, E. et al. - Lma política integrada de saúde para o Estado de São Paulo. Brasil. Rev. Saúde públ., S. Paulo, 9:169-79, 1975.

encontra bem próximo desse ideal é que poderá oferecer ao Estado sua colaboração plena na tarefa de desenvolvimento que a comunidade espera dele e dele exige. Este fato coloca a importância social que tem a atividade do Estado no campo da Saúde. De um lado, porque as atividades de proteção e recuperação da saúde constituem mecanismos válidos de redistribuição de renda; de outro lado, porque a ação governamental neste setor emerge da consciência de que o desenvolvimento nacional transcende o simples acúmulo de riquezas, compreendendo inovações sociais profundas e exigindo atuação prioritária fundada em uma programação flexível, coerente e conjugada de todos os agentes prestadores de serviços médico-sanitários.

Outro conceito de extrema importância a ser lembrado é de que a saúde é bem fundamental de todos os homens e de cada homem; por ser direito básico de cidadão, cabe ao Estado proporcionar todos os elementos necessários à sua manutenção e a seu desenvolvimento. Com esse objetivo, deve o poder público até mesmo procurar conscientizar a comunidade e seus componentes, individualmente, de que eles têm papel de relevo a desempenhar; mas, ao Estado caberá sempre a tarefa mais ampla de desenvolver atividades em áreas complementares à iniciativa particular e naquelas em que os investimentos exigidos forem de vulto que ultrapasse as possibilidades individuais.

Mas, existe ainda outro aspecto a ser considerado, ou seja, o fato de que o setor saúde é peça fundamental no conjunto de fatores que influenciam decisivamente o ritmo do desenvolvimento material da comunidade. $O$ que existe aquí é um círculo vicioso em que saúde e desenvolvimento se inter-relacionam, comportando-se como interdependentes. Trata-se de um exemplo de "causação circular", tal como definido por Myrdahl ${ }^{1}$.

De um lado, o baixo nível de desenvolvimento da comunidade, condicionando baixa renda individual e familiar, interfere com o nível de saúde do povo, por meio de diferentes mecanismos. Em primeiro lugar, pela redução de produção e da disponibilidade de alimentos. Em segundo lugar, pelas condições sanitárias deficientes, em especial no que se refere a saneamento básico (tratamento de água, deposição de excretas, coleta e destinação do lixo urbano) e às condições de habitação. Este fator é agravado pela exigência de elevados investimentos que devem ser feitos visando a solução do problema, principalmente em função do intenso ritmo de crescimento da população urbana. Em terceiro lugar, pela baixa renda familiar, de que decorrem baixas disponibilidades para alimentação, vestuário, habitação, etc. Em quarto lugar, pelo baixo nível educacional, com efeitos sobre a higiene individual, hábitos de saúde, etc.

Inversamente, o nivel de saúde e o grau de bem-estar geral da população interferem com o ritmo de desenvolvimento da comunidade, por meio de diversos fatores. Inicialmente, a quantidade e a natureza das enfermidades interferem sobre a capacidade de trabalho dos indivíduos, a qual representa componente fundamental da produtividade. A seguir, a ação sanitária, ao reduzir a mortalidade infantil, influi sobre a estrutura das idades e, portanto, sobre a quantidade de mão-de-obra total e a disponível. Depois, os níveis de saúde e bem-estar influem sobre a capacidade de consumo dos indivíduos. Por último, o elevado número de doentes exige altos investimentos em recursos curativos, reduzindo as disponibilidades relativas que o setor saúde pode destinar às medidas de medicina preventiva.

Um longo caminho deve ser ainda percorrido, antes que se possa atingir no Estado de São Paulo, como de resto no Brasil, o objetivo colocado pela definição da Organização Mundial da Saúde e que deve ser $o$ alvo do que se pretende com a caracterização de uma "política de saúde para o Estado de São Paulo". 
LIMA GONCALVES, E. et al. - Uma política integrada de saúde para o Estado de São Paulo, Brasil. Rev. Saúde públ., S. Paulo, 9:169-79, 1975.

Lm dos maiores obstáculos nesse longo caminho é representado pela total falta de coordenação que se observa no desenvolvimento das atividades dos diferentes setores-públicos e privados — de saúde: multiplicam-se as iniciativas paralelas que desgastam os recurso e escasseiam as iniciativas integradas, mesmo diante de situações que afligem a comunidade. Outro obstáculo é representado pela total desvinculação com que agem os órgãos que cuidam do saneamento básico, no que se refere à "autoridade sanitária", representada pela Secretaria de Estado da Saúde, como define o Decreto 52.182 de 1969 , cumprindo o artigo 134 da Constituição Estadual.

Em vista desses e de outros obstáculos, a consecução daquele objetivo geral supõe a colocação de alguns objetivos setoriais, que podem representar etapas intermediárias a serem cumpridas. Alguns desses objetivos são a seguir enumerados.

1 - Definição de um "sistema de saúde" a ser integrado por todas as estruturas públicas ou privadas que trabalham nessa área, com delimitação possível das atribuições que cabem a cada uma.

2 - Conscientizaçãs da comunidade a fim de que todos os recursos existentes sejam inseridos no esforço comum, que redundará em benefício de todos.

3 - Integração das estruturas que trabalham na área de saúde sob coordenação da "autoridade sanitária".

4 - Desenvolvimento dos instrumentos necessários à referida integração: legais, de coordenação e de controle.

5 - Capacitação da Secretaria da Saúde para desempenhar seu papel de "autoridade sanitária" em particular no que se refere à atualização institucional, à esestrutura organizacional e à preparação de quadros.

O primeiro objetivo setorial poderá ser viabilizado com a implantação do "sistema nacional de saúde", tal como figura no projeto de lei encaminhado pelo senhor Presidente da República ao Congresso $\mathrm{Na}$ cional, nos primeiros dias de fevereiro de 1975. Contudo, como a elaboração de nosso estudo precedeu à referida iniciativa do poder público federal, a proposição indicada continua integrando nosso quadro de proposições de uma "política de saúde", figurando aliás em primeiro lugar.

A conscientização da comunidade em relação aos problemas de saúde é objetivo extremamente importante, com vistas ao aumento da eficiência das medidas adotadas pelo poder público. Este pode e deve utilizar. em consequiencia, todos os meios e recursos a seu alcance, para buscar desenvolver a conscientização referida. Em trabalho recentemente publicado ", especialistas da Secretaria de Estado da Saúde de São Paulo definiram recursos que poderão ser utilizados com o objetivo de trabalhar na referida linha de conscientização da comunidade, especialmente pela utilização de meios de comunicação de massa e pelo emprego de recursos associativos. Sob este último ponto de vista, o estudo salienta o papel que poderia representar a estrutura formal da Igreja Católica.

O terceiro objetivo proposto é representado pela necessidade de integração das estruturas que trabalham na área de saúde, sob a coordenação da "autoridade sanitária" representada pela Secretaria de Estado da Saúde. Pela delicadeza que o assunto envolve, devem ser analisados com cuidado as proposições necessárias à integração no "sistema de saúde" das diferentes estruturas que trabalham na área, tal como foi sugerido no $30^{\circ}$ objetivo setorial proposto.

Como abordagem inicial, imaginamos que essa integração poderia assumir diferentes esquemas operacionais, dependendo do tipo de atividade exercida e do relacionamento administrativo atual e futuro. Basicamente poderia haver 4 tipos de integração: 1 . subordinação clássica; 2 . vinculaçāo; 3 . delegação; 4. coordena- 
LIMA GONCALVES, E. et al. - Uma politica integrada de saúde para o Estado de São Paulo. Brasil. Rev. Saúde públ., S. Paulo, 9:169-79, 1975.

ção, que distinguimos em coordenação funcional e coordenação de programas.

A subordinação clássica corresponderia à forma habitual de integração na área da administração, envolvendo o básico "princípio de hierarquia", ou seja, a relação de subordinação que une os órgãos do Poder Executivo, delimitando a autoridade ou a competência de cada um deles.

Como conseqüências desse "princípio de hierarquia" podem ser apontadas, dentre outras, as seguintes: 1. unidade de direção; 2. fiscalização ou controle do superior sobre o inferior; 3. substituição da autoridade inferior pela superior. caracterizando o instituto da "avocação"; 4. possibilidade de revisão, pela autoridade superior, dos atos praticados pela inferior; 5. aplicação de sanções; 6 . dever de obediência; 7 . delegação de atribuição pela autoridade superior.

A vinculação envolveria outro conceito jurídico básico que é o "princípio da tutela administrativa". Este define-se como o conjunto de poderes legais, embora limitados, que o Estado confere por lei aos órgãos centrais ou diretos a fim de exercerem ininterrupta vigilância ou controle sobre os atos das pessoas administrativas descentralizadas ou indiretas (autarquias, sociedades de economia mista, empresas públicas, fundações públicas) para garantir-lhes o mérito e a legalidade resguardando a consecução dos fins ou interesses coletivos.

A delegação de atribuições seria entendida como a transferência a outrem de tarefas que competem originariamente a determinada autoridade; a delegação é vedada, por preceito constitucional, entre estruturas integrantes de Poderes diferentes. A delegação corresponde a uma verdadeira transferência de competência: o órgão delegado toma a decisão, dentro dos poderes delegados, sob a forma habitual através da qual pratica os atos de sua própria competência. $\mathbf{O}$ órgão que age em virtude de uma delegação não se acha obrigado pelas ordens ou instruçôes do órgão delegante; os atos que assim praticar estão dentro da sua discrição, nos limites da norma mediante a qual se operou a delegação.

A coordenação suporia o desenvolvimento simultâneo e paralelo de todas as atividades administrativas do órgão coordenado por uma autoridade que exerce o papel de coordenador. $O$ que se distingue em nossas proposições é, de um lado. uma simples coordenação de programas, em que será necessário o prévio conhecimento dos planos de atividades de dois órgãos. com o objetivo de compatibilizar seu desenvolvimento e permitir que os resultados sejam otimizados. De outro lado estaria uma coordenação funcional em que a autoridade coordenadora poderá influenciar ou colorir, em determinada medida. as atividades inerentes a funcionamento da estrutura coordenada.

Com base na caracterização apresentada das diferentes figuras de integração de atividades, apresenta-se a seguir um quadro possível de redistribuição de estruturas e de atividades, com vistas à consecução do objetivo proposto.

\section{ANALISE DE ALGUNS PROBLEMAS PECULIARES}

A seguir tentamos ordenar a discussão de alguns tópicos referidos no quadro anterior, bem como alguns problemas mais relevantes.

1 - Por razões de ordem técnica e de otimização de rendimento, não se justifica a permanência no âmbito da Secretaria de Estado da Educação, do Serviço Dentário Escolar e do Serviço de Saúde Escolar; sua transferência para a Secretaria da Saúde é defendida inclusive pela maioria dos técnicos de educação.

2 - Quanto ao município de São Paulo, com toda a cautela e expectativa quanto à caracterização da Região Metropo- 


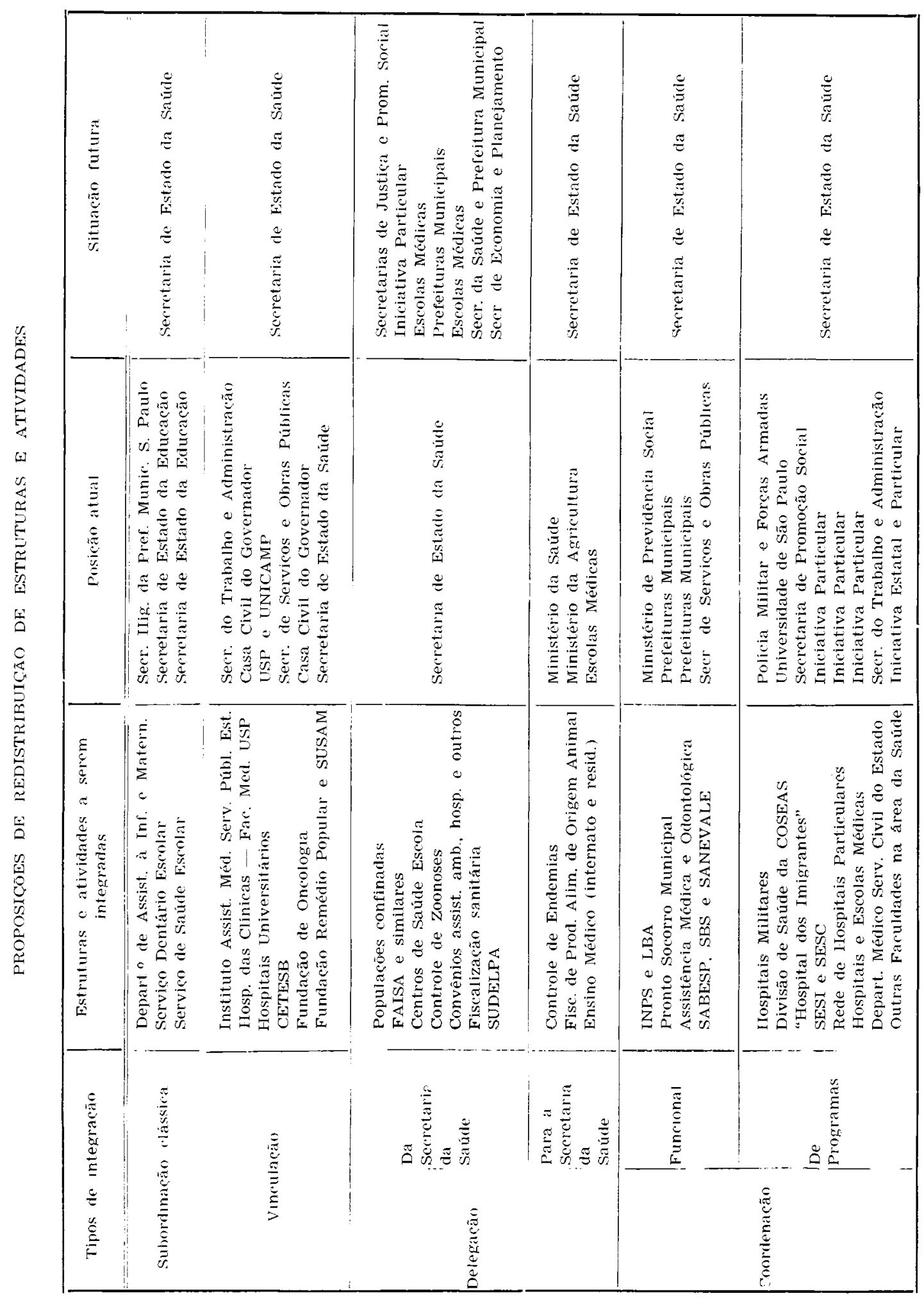


LIMA GONCALVES, E. et al. - Uma politica integrada de saúde para o Estado de São Paulo, Brasil. Rev. Saúde públ., S. Paulo, 9:169-79, 1975.

litana, parece haver possibilidade e conveniência de subordinar integralmente à Secretaria de Estado da Saúde os 57 postos do Departamento de Assistência à Infância e à Maternidade da Secretaria de Higiene da Prefeitura, a fim de melhor adequar uma rede unificada, em termos de distribuição do espaço, recursos humanos suficientes e pleno desenvolvimento das instalações. Essa passagem para o Estado permitiria a este até mesmo corrigir falhas de localização de suas unidades.

Quanto aos hospitais do Departamento de Higiene da Secretaria de Higiene da Prefeitura, devem ser enfatizados como retaguarda do Pronto Socorro Municipal. O Hospital do Servidor Público Municipal é parte do sistema previdenciário: sua "integração" seria feita por meio de indispensável coordenação de programas a serem desenvolvidos com a Secretaria de Higiene e Saúde da Prefeitura Municipal de São Paulo, à qual aquele Hospital está vinculado.

3 - Situação diferente é a representada pelo Instituto de Assistência Médica ao Servidor Público Estadual (IAMSPE) e Hospital do Servidor Público Estadual "Francisco Morato de Oliveira". Aqui, um equipamento eminentemente de saúde permanece vinculado à estrutura de recursos humanos do Estado, o que representa básica distorção dos objetivos fundamentais e finais da própria instituição. 0 exemplo da Guanabara, onde o Instituto de Aposentadoria dos Servidores do Estado da Gauanabara (IASEG) é vinculado à Secretaria da Saúde, demonstra a eficiência que proposição semelhante em São Paulo conferiria à área geral de prestação de assistência hospitalar.

4 - Problema delicado é o representado pelos hospitais-escola, cujo exemp'o mais típico é o Hospital das Clínicas da Faculdade de Medicina da Universidade de São Paulo. A título de conferir-lhe maior flexibilidade, a instituição foi transformada em autarquia e vinculada à $\mathrm{Ca}$ - sa Civil do Governador. Trata-se de estruturada onde se desenvolvem simultaneamente atividades de tipo assistencial e de tipo docente. A atual vinculação acarretou considerável hipertrofia do setor assistencial, em detrimento das atividades de ensino. Mas, além disso, deu oportunidade a outras distorções: atendimento prioritário das necessidades de ampliação física das instalações, em detrimento de aspectos relativos a recursos humanos, sempre vinculados a número considerável de restriçōes de ordem administrativa; entrosamento limitado a "autoridade sanitária", mesmo em situações assistenciais aflitivas para a comunidade paulista; desenvolvimento de um sentido exagerado de "centro universitário de administração hospitalar", provavelmente em decorrência do reduzido número de estruturas atuantes na área. mas que acarreta conseqüências relevantes - criação de um instrumento de desenvolvimento de "know-how" administrativo em paralelo com o que deve existir nos órgãos do Estado, especificamente destinados a essa atividade, que são os institutos universitários; substituição de setores nacionais de consultoria médico-hospitalar disponíveis.

A integração de hospitais-escola desse tipo à Secretaria da Saúde permitia o desenvolvimento de sua atividade assistencial de maneira equilibrada, ao lado de abrir para a Faculdade de Medicina rica hipótese de trabalho junto aos órgãos da comunidade. E importante lembrar aqui todo o empenho que o moderno ensino médico vem colocando em termos de inserir-se o mais próximo possível dos usuários; junto à sua família, à sua casa, ao seu "em torno". Tudo isso seria facilitado, uma vez que a Secretaria da Saúde é que detém os instrumentos de abordagem direta da comunidade, que são as unidades sanitárias.

Outra linha de argumentos refere-se àquilo que hoje já é condição para o desenvolvimento do ensino médico e, por 
LIMA GONÇALVES, E. et al. - Uma politica integrada de saúde para o Estado de são Paulo, Brasil. Rev. Saude pübl., S. Paulo, 9:169-79, 1975.

conseguinte, da própria assistência médica em São Paulo. Trata-se de inserção da Secretaria da Saúde, representada por seus hospitais próprios, nas atividades de ensino médico, essencialmente as de internato e residência. $O$ proveito seria das escolas de medicina e dos hospitais inseridos no programa, pela inevitável elevação de seu nível assistencial, em decorrência da presença de jovens internos e residentes. Nesse sentido, a experiência que foi iniciada há 3 anos nos hospitais próprios do Instituto Nacional de Previdência Social (INPS) em São Paulo é muito esclarecedora.

Os mesmos argumentos aqui colocados, para justificar a vinculação do Hospital das Clínicas à Secretaria da Saúde, valem para defender a mesma tese aplicada aos futuros "hospitais universitários", o da Cidade Universitária "Armando de Salles Oliveira", o de Ribeirão Preto, ambos vinculados à Úniversidade de São Paulo, bem como o de Campinas, vinculado à Universidade de Campinas (Unicamp).

5 - Por razões óbvias de natureza técnico-científica, a Fundação de Oncologia criada recentemente, deveria ter sua vinculação transferida da Casa Civil do Governador para a Secretaria da Saúde.

6 - Os serviços médicos de populaçōes confinadas ou recolhidas não deveriam ser assumidos pela Secretaria da Saúde; o que se poderia prever é uma espécie de obrigatoriedade de registro, nessa Secretaria, do profissional médico responsável por essa população, o qual receberia supervisão do sanitarista mais próximo para a realização obrigatória de programas preventivos para essas populações.

7 - As atividades de vigilância e de poder de polícia sanitária deveriam ser assumidos pela Secretaria, exceção feita à polícia sanitária de portos e fronteiras internacionais, que é competência do governo federal. Assim, seria transferido para a Secretaria o convênio do Ministério da Agricultura com a Divisão de
Inspeção de Produtos Alimentícios de Origem Animal (D.I.P.A.O.A.) e estudado em cada caso, para credenciamento do corpo de engenheiros, a delegação às Prefeituras Municipais para aprovação de plantas e outros aspectos de engenharia sanitária atualmente concorrentes com o Estado.

8 - Em diferentes situaçōes, seria perfeitamente possível delegar a instituiçōes particulares a execução de certas tarefas que as unidades da Secretaria da Saúde desempenham habitualmente. Exemplo é, entre outros, a Fundação de Assistência à Infância de Santo André, cujo nível de atuação é dos mais significativos.

9 - Delegação semelhante poderia ser feita em relação a entidades oficiais; é o que já se faz com a Superintendência do Desenvolvimento do Litoral Paulista (SUDELPA).

10 - Igual tipo de delegação caberia em relação aos Centros de Saúde Escola, operados já por algumas entidades de ensino médico. E o que acontece também com convênios desinados a assistência ambulatorial e hospitalar.

11 - Inversamente deveriam ser delegadas à Secretaria da Saúde certas atividades específicas das escolas médicas, no referente a programas de internato e residência, a serem desenvolvidos nos hospitais da Secretaria, como já se disse.

12 - Às Prefeituras Municipais poderia ser delegada a competência no controle de zoonoses, tal como é feito atualmente, de maneira não definida legalmente no Município de São Paulo.

13 - Definição legal deveria merecer também o trabalho atualmente desenvolvido pela Secretaria da Saúde no controle de endemias, atribuição específica de órgãos federais.

14. - Abrir uma frente ampla de assistência médico-hospitalar envolve a necessidade de enfrentar uma situação-problema carente de definição institucional e ainda sem dimensões precisas. A assistência médica geral supletiva e a especializada para tisiologia, hanseniologia, psi- 
LIMA GONÇALVES, E. et al. - Uma política integrada de saúde para o Estado de são Paulo. Brasil. Rev. Saúde pübl., S. Paulo, 9:169-79, 1975.

quiatria, etc. tal como o Estado vem mantendo, parece ser o adequado, desde que possa ser reforçada por um dispositivo financeiro que permila, eventualmente, abrir os leitos para o INPS e Legião Brasileira de Assistência (LBA) ou outras instituições. Esse é o enfoque que vem sendo adotado para a cessão de instalações das Unidades sanitárias da Secretaria através do Convênio com a Previdência Social que criou o CIAM (Centro de Integração de Atividades Médicas).

Esta iniciativa envolveria, certamente, a necessidade de um amplo entendimento com o alto escalão administrativo federal, mas permitiria o desenvolvimento de um programa integrado de saúde em São Paulo, capaz de enfrentar as deficiências que marcam até hoje a atividade da estrutura previdenciária. Não é necessário salientar que esse programa teria importância nacional, como experiência de extraordinária relevância. Obviamente exigiria todo um trabalho de detalhamento, até nível local, para o qual já existem subsídios suficientes.

15 - Na mesma linha de coordenação funcional deveriam ser inseridas no "sistema de saúde" as atividades do Pronto Socorro Municipal e dos Serviços de Assistência Médica e Odontológica mantidos por numerosas Prefeituras Municipais.

16 - Os problemas de saneamento do meio merecem consideraçōes amplas e cuidadosas. De um lado, é indiscutível que a Secretaria da Saúde deve absterse de qualquer ação executiva de vulto, direta ou indireta. na área de saneamento e engenharia sanitária. Mas, não pode, de outro lado, permanecer marginalizada das decisões gerais + das definições de prioridades na área.

Diga-se inicialmente que a fixação da política básica de saúde e, portanto, de saneamento deveria ser atrihuição específica da Secretaria de Estado da Saúde com o assessoramento de alto nível do Conselho Estadual de Saúde e dos órgãos técnicos de nivel central. Assim. a defi- nição de prioridades de atividades, obras e serviços de saneamento, no âmbito da administração estadual, deveria ser atribuição privativa dos órgãos de cúpula da área, dos quais participaria a Secretaria da Saúde. A razão fundamental é que a tarefa básica de fixação da política e das prioridades neste campo deveria constituir prerrogativa de quem deteria o comando unificado na área.

As atividades de "planejar, executar e operar serviços de saneamento básico em todo o território do Estado de São Paulo, compreendendo a captação, tratamento e distribuição de água e a coleta, tratamento e disposição final de esgotos" estão previstas no artigo $1 .^{\circ}$ da Lei $n .^{\circ} 119 / 73$. que autorizou a constituição da Companhia de Saneamento Básico do Estado de São Paulo (SABESP). A integração com esta área deveria ser feita em nível de coordenação funcional, o mesmo devendo ser dito quanto ao Saneamento da Baixada Santista e a Companhia Regional de Águas e Esgotos do Vale do Ribeira (SANEVALE). Para tanto, seria indispensável alterar certos artigos dos estatutos da SABESP, a fim de que o principal órgão executor dos programas de saneamento básico do Estado deva ouvir a "autoridade sanitária", além das autoridades a que já deve submeter atualmente seus programas, representados pelas Secretarias de Estado de Serviços e Obras Públicas, de Economia e Planejamento e da Fazenda.

Além disso, dada a importância das moléstias de veiculação hídrica no quadro nosológico do Estado, cabe ao poder público exercer rigoroso controle das águas de abastecimento público ou privado, assim como os problemas gerais de contaminação; grande parte desse controle é feito atualmente pela Companhia Estadual de Tecnologia de Saneamento Básico e de Controle de Poluição das Águas (CETESB).

Para corrigir a situação atual a alternativa mais eficiente seria vincular à Secretaria da Saúde os órgãos que permitem a execução da avaliação de desem- 
LIMA GONCALVES, E. et al, - Uma politica integrada de saúde para o Estado de São Paulo. Brasil. Rev. Saúde puibl., S. Paulo, 9:169-79, 1975.

penho técnico das estruturas que devem trabalhar no campo de saneamento. Esses órgãos são a Superintendência do Saneamento Ambiental (SUSAM), atualmente já vinculada à Secretaria e a CETESB, atualmente integrada à Secretaria de Serviços e Obras Públicas. 0 exame dos estatutos desta Companhia revela sua dependência exclusiva da referida Secretaria, bem como das Secretarias da Economia e Planejamento e da Fazenda, agora quanto a aspectos orçamentários; trata-se de situação que precisaria ser corrigida.

No âmbito interno da Secretaria, dada a multiplicidade de variáveis e de um número ainda maior de incógnitas quanto às características institucionais da Grande São Paulo, seria recomendável que a área de atuação atual do Departamento de Saneamento da Coordenadoria de Saúde da Comunidade viesse a constituir uma Assessoria do Secretário em Saneamento e Engenharia Ambiental, com finalidade de ir medindo as repercussões para os níveis de saúde, do funcionamento de outros órgãos de todos os níveis e esferas do Governo.

Com relação à administração descentralizada (SUSAM e CACESQ -- Campanha de Combate à Esquistossomose) talvez fosse preferível utilizar como critério para eventual separação na administração descentralizada, aquelas atividades que envolvem ações sobre o meio conjuntamente com açōes curativas, daquelas atividades em que as ações se exercem somente sobre o meio.

17 - Atividade de policiamento da alimentação pública deveria ser distribuída. na região da Grande. São Paulo, entre as unidades sanitárias, a exemplo do que acontece com o resto do Estado. Deve ser lembrada aqui a coordenação com o pessoal de fiscalização sanitária que trabalha nas Administrações Regionais da Prefeitura de São Paulo.

18 - E presumivel que profundas alterações venham a ocorrer na divisão tradicional de funções já agora concorrentes - conflitantes em muitos casos entre as diversas esferas do governo, a fim de situar claramente a competência da Região Metropolitana. Este enfoque poderá levar a um novo grupamento de funçōes, inclusive na área da saúde.

19 - Deveriam ser excluídas desde logo as atividades que as Unidades Sanitárias da Secretaria vêm desenvolvendo por imposição externa, tais como os exames de motoristas e as inspeções médicas para ingressos ou licenças de servidores; caso sejam mantidas, devem sê-lo exclusivamente em locais onde não haja outra agência ou possibilidade de atendimento e devem ser remuneradas ou atendidas mediante reforço de pessoal.

20 - Amplo trabalho de coordenação de programas deveria ser desenvolvido em relação à rede particular, em estruturas destinadas sobretudo à assistência médico-hospitalar. Trata-se de iniciativas com finalidade lucrativa algumas, de cunho filantrópico, outras. Todas merecem apoio e consideração; sua irtegração em programas globais de assistência médico-hospitalar deveria incluir a obtenção, em contrapartida, de dados regulares sobre morbidade, bem como a garantia de desenvolvimento de programas preventivos de imunização e outros, de acordo com a orientação da Secretaria.

Trabalho semelhante de coordenação de programas deveria ser desenvolvido com outras entidades hospitalares ou assistenciais vinculadas a diferentes estruturas oficiais ou particulares. E o que acontece com os hospitais da Polícia Militar do Estado de Sáo Paulo e das Forças Armadas; com o chamado "Hospital dos Imigrantes" da Secretaria de Estado da Promoção Social; com a Divisão de Saúde da Coordenadoria de Saúde e Assistência Social (COSEAS), integrada na Ul.iversidade de São Paulo; com o Departamento Médico do Serviço Civil do Estado da Secretaria de Trabalho e Administração; com o Serviço Social da Indústria (SESI) e o Serviço Social do Comércio (SESC) ; com as Escolas Médicas e com outras Faculdades que ope- 
LIMA GONÇALVES, E. et al. - Uma política integrada de saúde para o Estado de sáo Paulo, Brasil. Rev. Saúde públ., S. Paulo, 9:169-79, 1975.

ram na área da Saúde, sejam as dependentes de órgãos estatais, sejam aquelas ligadas à iniciativa particular.

21 - A preocupação com uma adequada nutrição vem sendo sentida em todos os niveis de Governo e sensibilizando a comunidade, pelos problemas que suas falhas vêm mostrando. Vários órgãos e entidades vêm desenvolvendo atividades nesse sentido, limitadas, porém, seja a segmentos da população sob sua responsabilidade eventual, seja à disponibilidade de recursos financeiros e ação inter-setorial capazes de desenvolver programas nutricionais que ultrapasse o período e o espaço dessa responsabilidade. Exemplos são escolas, unidades sanitárias, hospitais, presídios, etc. Também se identificam grupos altamente vulneráveis que têm permanecido a descoberto, enquanto não se localizam como clientela daquelas unidades e estejam com condições agravadas por fatores sócio-econômicos-regionais. Assim, se há um grupo pré ou extra-escolar, ou crianças em férias, ou gestantes rnarginalizadas ou pessoas jovens em situação de abandono, etc. as preocupações com adequada nutrição, não os alcança.

Neste terreno à Secretaria da Saúde poderia ser atribuída a coordenação na àrea geográfica do Estado, para se obter um dimensionamento exato de problema e o acionamento ordenado de ação para uma continuidade de programas e a suplementação necessária para total cobertura. A coordenação seria funcional, sbrangendo a distribuição de recursos financeiros através de órgão colegiado ou com representabilidade funcional, vinculado à Secretaria da Saúde ou, ainda, através de uma clara e precisa destinação orçamentária comandada pela Secretaria. Seriam mantidas as atuais subordinaçōes de atividade desenvolvidas com populações confinadas e localizadas bem como sobre a clientela das unidades Sanitárias.

Está visto que o desenvolvimento de um programa tão amplo e tão complexo como o proposto exigiria uma série de medidas preliminares, que represerttam os dois úl- timos objetivos setoriais dentro do conjunto que estamos analisando. Trata-se, na verdade, mais de objetivos-meios capazes de possibilitar a consecução daqueles que são os objetivos-fins; estes correspondem aos procedimentos de integração que foram analisados.

O primeiro dos objetivos-meios referidos seria o desenvolvimento dos instrumentos necessários à integração descrita, tanto os instrumentos legais, como os instrumentos estruturais de coordenação e controle.

O último objetivo-meio seria a capacitação da Secretaria da Saúde para assumir com eficiência o papel central de "autoridade sanitária", que teria de desempenhar.

Último dado básico que deve ser posto em evidência: o esforço maior a ser desenvolvido quando se visa à saúde da comunidade não pode prender-se a conceitos parciais de atendimento, exatamente porque a saúde é um todo global. É indiscutível que se justificam amplamente iniciativas no campo da medicina preventiva, como programas permanentes de imunização, porque parcela muito considerável de óbitos ocorridos deve-se ainda a doenças transmissíveis, controláveis ou erradicáveis pela vacinação: poliomielite, tuberculose, raiva, tétano, difteria, coqueluche e sarampo entre outras. Mas, é também indiscutível que têm que merecer igualmente muito interesse os problemas de medicina curativa, de que muito carece a população paulista, não apenas no que se refere ao acesso a ambulatórios e consultórios, para entrevista médica e confirmação de diagnóstico, como também no que tange ao tratamento consequente, seja de tipo medicamentoso, pela assistência farmacêutica adequada, seja de tipo cirúrgico, pela garantia de internação hospitalar suficiente.

Está visto que a responsabilidade das medidas de caráter preventivo caberão sempre integralmente ao poder público, ao passo que, no campo da medicina curativa, caberia distinguir a clientela sob 
LIMA GONCALVES, E. et al. - Uma politica Integrada de saúde para o Estado de São Paulo, Brasil. Rev. Saude puibl., S. Paulo, 9:169-79, 1975.

regime de gratuidade da que pode participar do custeio e daquela que deve arcar integralmente com os custos assistenciais. Nos dois primeiros casos, o custeio caberá basicamente ao poder público, seja fede- ral, seja estadual ou municipal. $O$ que importa é que todo o conjunto de órgãos ou estruturas que trabalham na área da saúde sejam integradas num sistema de funcionamento harmonioso e coerente.

RSPU-B/257

Lima Gonçalves, E, et al. - [A comprehensive health policy for the State of S. Paulo, Brazil]. Rev. Saúde públ., S. Paulo, $9: 169-79,1975$.

SUMmary: A health polioy for providing attention for the population of the Brazilian State of S. Paulo, based on a threefold program of integration of facilities is proposed: the integration of preventive and healing medical activities; the integration of the sanitation authorities, under a central health authority, represented by the State Department of Health; the integration, in a true "health system", of the federal, state and private out-patient and inpatient clinics.

UNITERMs: Health. Resource integration. S. Paulo, Brazil. Health System.

\section{REFERENCIAS BIBLIOGRAFICAS}

1. MYRDAHL, G. -- Perspectivas de uma economia internacional. Rio de Janeiro, Ed. Saga, 1967.

2. SÃo PAULO. Secretaría de Estado da Saúde - Caracterização da clientela da Divisão de Saúde - São Paulo Centro. São Paulo, 1973.

Recebido para publicaç̃o em 25-02-1975 Aprovado para publicacão em 04-04-1975 